\title{
Decoding COVID-19: What Dentist Should Know?
}

\author{
Pudi Sriharsha $^{1}$, Naseemoon Shaik ${ }^{2}$, Supriya Yadlapati ${ }^{3}$, Pranav S ${ }^{4}$ \\ ${ }^{1}$ Assistant Professor, Department of Prosthodontics and Crown \& Bridge, \\ ${ }^{2}$ Assistant Professor, Department of Pediatric Dentistry, ${ }^{3,4}$ Final Year BDS Student, \\ MNR Dental College and Hospital, Sangareddy, Hyderabad \\ Corresponding Author: Naseemoon Shaik
}

\begin{abstract}
The outbreak of the Corona virus has rapidly escalated globally as a Pandemic. Due to the characteristic dental setup \& procedures the risk of cross infection is sky high not only to the Dental health care personnel but also to the patients. During this time utilizing digital resources to assist patients can be of great advantage. Doing less invasive procedures with more protection and screening can be the finest way to battle the situation. This pandemic situation provides an unprecedented opportunity for the dental community to shift the practice towards the use of Teledentistry. Teledentistry may overcome the obstacle in providing sufficient oral health services comfortably during the current pandemic situation.

Although the world of Dentistry has many challenges the main aim is to provide valuebased dental care and exploit the tremendous potential of Telecommunication services \& other virtual and digital alternatives to confront the challenges that are faced due to infection control, lack of awareness etc.
\end{abstract}

Keywords: Artificial intelligence, COVID-19, Dentistry, Pandemic, Teledentistry.

\section{INTRODUCTION}

Devastation by a deadly virus, these words make sense since the outbreak of the deadly Coronavirus, but as impossible as it seems, it does have a major impact on the Human society now. Humans have been a dominant species for over thousands of years now leading to mass

Destruction, War, Pollution etc \& that We are the cause. But like all living organisms, the earth unleashed a fever to fight this infection. Viruses are commonly a part of Earth's natural ecosystem. It becomes a pandemic when it affects a large scale of population with a high Mortality Rate. Many Pandemics were seen by the world before the Covid Virus.

\section{HISTORY}

While some of the earliest pandemics faded by obliterating out parts of the population, some were averted to an extent with the onset of medical advancements and public health initiatives which were able to terminate the spread of other deadly sickness (Dave Roos,2020).

As the waves of disease washed again and again over the human population, it took centuries for science to fully understand the nature of invisible world of microbes. Some tried to explain it through theories while others blamed the beliefs. There is no wonder that as human civilizations flourished so did the spread of Infectious agents. The most deadly Pandemics which the World had faced were:

1. Plague of Justinian which was caused by Yersenia pestis is considered one of the deadliest pandemics in the history.

2. Black Death, the Bubonic plague where people had no idea on how to control it \& invented Quarantine which they thought that it's the only way to fight the pandemic.

3. The Great Plague of London

4. Smallpox also created a havoc among the population which later became the first virus epidemic to be terminated by a vaccine. 
5. Cholera, a bacterial disease killing tens of thousands of people. The prevailing scientific theory of the day said that the disease was spread through evil air which they termed as "miasma."

Not only these, but Antonine Plague, Cyprian Plague, Leprosy, Fiji Measles Pandemic, Russian Flu, Spanish Flu, Asian Flu, HIV/AIDS,SARS virus whose advent dates back to 2003,along with the present day COVID 19 virus which is creating a havoc among the masses.

People previously had no real understanding of how to fight the pandemic, other than trying to avoid sick people, but now with the advancement of science \& Technology it can be managed to an extent by taking a set of precautions.

\section{COVID-19}

COVID-19 is caused by infection due to Severe Acute Respiratory Syndrome Coronavirus 2 (SARS-CoV 2). The outburst of Corona virus disease occurred in 2019 (COVID-19), which first began in Wuhan, China, in the late 2019, which has spread rapidly to 205 countries as of March 30, 2020, and has been officially declared a global pandemic by the World Health Organization (WHO) (Cucinotta D and Vanelli M, 2020).

\section{Clinical Relevance}

COVID-19 has a high infectivity rate, primarily due to its spread through respiratory droplets.

It has an incubation period of 1-14 days, after which clinical symptoms such as fever, cough, fatigue, sputum production, shortness of breath, sore throat, and headache start to manifest. Loss of smell is a very strong indicator of Covid infection (Elshazli RM et al., 2021)

It affects patients of all ages with varying levels of severity. It has also been shown to have cutaneous manifestations called Covid-19 exanthems which include maculopapular, urticarial, vesicular rashes, chilblain-like petechiae. Maculopapular being the most common form (Singh $\mathrm{H}$ et al., 2020, Zhao Q et al., 2020).

\section{NEW STRAINS}

A new double mutated strain of SARS CoV2 has been detected as the main culprit in second wave. Brazilian, South African and UK strains were also observed $\&$ identified. Eminent public health researcher Peter Daszak and his team have estimated that there are as many as 5,000 coronavirus strains globally waiting to be discovered. India's second wave is now considered the worst COVID-19 surge in the world (Xu S and Li Y, 2020).

The second wave for the US started in 2020 in mid-June and reached its peak during the fourth week of July. On the other hand, the second wave in India started at the end of February in 2021. According to Ministry of Health \& Family Welfare, on 10 February, at the start of the second wave, India confirmed 11,000 cases $\&$ in the next 50 days, the daily average was around 22,000 cases. But in the following 2 weeks, cases rose sharply with a daily average reaching 89,800 cases.

These mutated viruses have the potential to skip the immunity and even Vaccine. This is the reason for increase in the re-infection cases \& cases among vaccinated people. It is considered to be more Infectious than the previous Covid Strain affecting the younger population along with many added symptoms like Hearing problems, Conjunctivitis.

\section{TRANSMISSION}

Transmission is mainly by contact and droplet transmission (due to actions like Sneezing, coughing, singing, talking), Airborne transmission (aerosol generating procedures), Fomite transmission (contaminated surfaces). Although, animal to human transmission was considered to be the prime mode of transmission, it has now been realized that symptomatic or asymptomatic infected patients, are the main reason for high infectivity (Peng X et al., 2020). Current evidence clearly stated that person to person transmission occurs mainly via droplet spread. (Abd EW et al., 2020) 


\section{PREVENTION STRATEGIES}

- Awareness \& Proper Education is a major criteria which aids in preventing the disease from further spread.

- As the contamination occurs through respiratory droplet infection it requires close contact to continue its spread. Therefore, maintenance of Social Distancing is a must (Bahl P et al., 2020).

- By wearing a mask when physical distancing is not possible. Masks can help prevent the spread of the virus (Sommerstein R et al., 2020).

- By not touching eyes, nose or mouth.

- Maintain a safe distance from anyone.

- Often cleaning the hands using soap , water or alcohol based Rub(Sanitizer)

- Vaccination has to be taken.

\section{COVID \& DENTISTRY}

\section{Infection control and Safety Challenges in} dentistry:

The Corona virus is known to be thoroughly transmissible when patients are most symptomatic, transmission can even occur before any symptoms or signs are evident. Additionally, the majority of people infected with SARS-CoV-2 may have no symptoms or symptoms that resemble seasonal allergies or influenza, contributing to a significant number of undiagnosed cases. Hence, even asymptomatic dental patients should be considered as potential carriers and therefore it is recommended to implement a routine screening process for both patients and staff, to ensure that both symptomatic and recently exposed individuals stay home for the safety of everyone (Coulthard P, 2020)

In a dental setup, in addition to droplets, procedures on patients entailing the use of high-speed handpiece or ultrasonic instruments may cause their saliva, secretions or blood to aerosolize the viral particles into the surroundings. The risk of cross-infection could be high between dental practitioners and patients owing to its complexity in dental settings and aerosol involvement which occurs during procedures.

Strict infection control and sterilization protocols have to be followed in order to; practice and research ethics, emergency protocols, teledentistry \& dental practice management are essential factors to be considered during practice (Parin Bhanushali, 2020)

\section{ACCORDING TO IDA (Indian Dental Association) \& CDC (Centers for Disease Control \& Prevention) PROTOCOL COVID-19, 2020}

\section{Dental Emergencies}

Potentially life threatening conditions which require urgent and immediate treatment to alleviate severe pain or infection which includes the following:-

i. Uncontrolled bleeding \& Pain.

ii. Cellulitis or a diffuse soft tissue bacterial swelling.

iii. Infection with intra oral or extra oral swelling that potentially compromises the airway.

iv. Trauma involving the face or the facial bones.

v. Severe trismus

vi. Persistent non healing ulcers

Urgent Dental Care is required during the following Conditions:-

$>$ Severe dental pain from pulpal inflammation

$>$ Pericoronitis

$>$ Dry socket

$>$ Abscess or localised pain and swelling

$>$ Tooth fracture

$>$ Dental trauma with avulsion/ luxation

$>$ Restorations causing severe pain

$>$ Suture removal

$>$ Orthodontic wires or appliances piercing or impinging or causing trauma to oral mucosa

$>$ Biopsy of abnormal tissue.

The current treatment guidelines to be followed for the elective care of the patient as well as for the Safety of the Dentist. 


\section{Preventive Safety measures include}

- According to the possibility, by performing procedures in a negative pressure room, (airborne infection isolation room or AIIR) for aerosolgenerating dental procedures.

- Consider taking extraoral radiographs instead of intraoral (e.g. panoramic radiography) to avoid gag reflex.

- By placing a portable HEPA air filtration unit within the vicinity of the patient's chair.

- By limiting the number of visitors and dental healthcare providers during the procedure.

- By using a dental hand-piece which has an anti-retraction function.

- By practising four-handed dentistry.

- Minimise the use of high evacuation suction and rubber dams to minimize droplet splatter and aerosol generation.

- Reduce the use of ultrasonic instruments and 3-way syringes.

- Performing endodontic procedures with dilute solutions of sodium hypochlorite $(1 \%)$.

- Use resorbable sutures

- Disinfect surfaces with EPA-approved chemicals to maintain a dry, clean environment.

- Wear an N95 or equivalent mask or higher-level respirator such as a disposable filtering face piece respirator, a powered air-purifying respirator or an elastomeric respirator, eye protection (like goggles, face shields),gloves etc can be used (Balaji SM 2020, Bhanushali P 2020).

The current impact of Covid is not only on the Patient but also affecting the Economic status of the Dental Practice owners. (Puneet Bhatnagar,2020)

New ideas which can be followed for a better understanding \& a smooth run of Dental Practice include

- Patient management-by informing patients about a particular time slot allotted for conducting the procedure.
- Tele Screening has to be done by evaluating the patient initially. Patient screening protocols have to be strictly enforced.

- Patients Travel history has to be checked upon before the treatment.

- Efficient Staff Management is the key for a better Dental Practice.

- Cases which require urgent care(Acute pain, Swelling) can be treated but with utter care \& strict safety standards.

- Pharmacological management by using Antibiotics \& Analgesics should be able to control the risk of exposure to the infection.

- Close follow up can be done by using telephone or video conferencing.

\section{TELEDENTISTRY A BOON IN THE FUTURE OF DENTISTRY}

New approaches such as Teledentistry will help dentists assist patients without summing up the risk of cross infection. It is a perfect affair which can strike equilibrium between safety of the health care professionals yet providing optimum dental care to the patients requiring emergency intervention. (Sikka N, 2020) Teledentistry can be of great aid in this current pandemic situation. Teleconsultation, telediagnosis, teletriage, and telemonitoring are subunits of teledentistry.

To engage in tele-dentistry, the patient must need a smartphone with Internet connection. For the dentist, a cloudbased teledentistry platform which can support both real-time streaming of videos $\&$ "store and forward" of clinical data collected, including data from the electronic health record (EHR) and photos received from the patient. Such a platform is able to aggregate all data allowing the dentist to remotely evaluate the patient and develop recommendations (Jyoti Prajapat,2021).

Services provided as part of a teledentistry may include problem-focused and urgent care evaluations (e.g., acute swelling/pain) and guiding the patients with pharmacologic management; follow-up care 
after emergency visits; and virtually triaging of patients for COVID-19 symptoms (Singh N 2020).

The entire process of networking, sharing digital information, distant consultations, workup and analysis is dealt just by a click on the computer or mobile screen.

\section{ARTIFICIAL INTELLIGENCE (A.I.)}

With the advancement of Science and Technology, Dentists now employ a A.I. software to analyse health data, research findings and treatment techniques. This greatly helps to gain more insights in clinical decision making to their patients. They can not only choose better treatment options and possibilities but also help the clinician in understanding each case with personalized care.

\section{AUGMENTED REALITY (AR)}

It is done using mobile or web programming. It has the ability to simplify procedures and reduce operative time. The patient is presented with immediate visual representation of complete treatment procedure he/she is going to receive. The mechanism of how it works is by matching a 3D scan of the patient's oral cavity to the scans of normal teeth. Once the software stores the data related to patient's teeth, it overlays the improved teeth \& thereby a "virtual mirror" is offered to the patient which helps the patient to understand his condition better.

The significant application of AR is its use in prosthodontics, implantology, esthetic planning etc. This type of technology holds a paramount importance, especially during this pandemic time where the patient is able to analyze \& understand his own condition.

\section{VIRTUAL REALITY (VR)}

Its main aim is to guide dental students \& professionals to immerse in a virtual environment just by using a headset \& VR goggles which helps them not only tackle complicated cases but also affects their performance during the challenging procedures they may face in the future.

It helps to stream operations live for those who are unable to peek over the shoulder of a surgeon when the procedure is held.

\section{COMPUTER-ASSISTED DESIGN (CAD) \& COMPUTER ASSISTED MANUFACTURE (CAM)}

This is not a 21 century mantra but rather used since a long time now. 3Dprinting is done just by taking a picture of the tooth \& relayed in the machine, which builds a crown in the Dental office itself. This kind of technique especially helps during the Covid Pandemic period where the manual modeling \& inclusion of dental labs can be eliminated.

\section{INTRA ORAL CAMERA}

This eliminates the problem of the Dentist to peep inside the patient's mouth for a better view of the problem. The intraoral cameras are a perfect remedy to the problem which is uncomfortable to the patient as well as the Dentist, especially during the pandemic time when maintaining a distance from the patient is recommended.

\section{CONCLUSION}

Carefulness while working with patients is the key for the safety \& success of the Dentist.

Strict protocols are to be followed along with formulation of new guidelines with which fighting Covid wouldn't be an uphill battle.

Especially at these times where the viral strains are constantly changing \& Vaccination requires a lot of research \& study. With time, as still the research is going on, to identify \& exterminate the virus it is our duty to be cautious of our actions.

Therefore, these kinds of technologies which were once considered futile as they were high priced to practice in Dentistry can now be reached. Advent of these services will prove to be an effective weapon in diagnosis \& treatment planning of various conditions which once required heavy manual \& laboratory work. With the 
availability of newer technologies, treatment recommendations let us hope that in days to come, Dentistry will emerge successfully as it has done in the past. The most important thing about this pandemic episode is that how and what we learn- as individuals, as students \& as Dental practitioners.

\section{Acknowledgement: None}

\section{Conflict of Interest: None}

\section{Source of Funding: None}

\section{REFERENCES}

1. Abd EW, Eassa SM, Metwally M, AlHraishawi H, Omar SR. (2020). SARSCoV-2 Transmission Channels: A Review of the Literature. MEDICC Rev.,22(4):5169. PMID: 33295321.

2. Bahl, P., Doolan, C., de Silva, C., Chughtai, A. A., Bourouiba, L., \& MacIntyre, C. R. (2020). Airborne or droplet precautions for health workers treating COVID-19?. The Journal of infectious diseases, jiaa189. Advance online publication. https://doi.org/10.1093/infdis/jiaa189

3. Balaji SM. (2020).COVID-19-Future of dentistry. Indian J Dent Res, 31:167-8.

4. Bhanushali P, Katge F, Deshpande S, Chimata VK, Shetty S, Pradhan D. (2020).COVID-19: Changing Trends and Its Impact on Future of Dentistry. Int J Dent. May 29;2020:8817424. doi: 10.1155/2020/ 8817424. PMID: 32565812; PMCID: PMC7260644.

5. Centers for Disease Control and Prevention. (2016). Summary of Infection Prevention Practices in Dental Settings: Basic Expectations for Safe Care. Atlanta, GA: Centers for Disease Control and Prevention, US Dept of Health and Human Services; http://www.cdc.gov/hai/settings/outpatient/o utpatient-care-guidelines.html

6. Coulthard P. (2020). Dentistry and coronavirus (COVID-19)-moral decisionmaking. Br Dent J., 228(7):503-505. doi: 10.1038/s41415-020-1482-1. PMID: 32277203.

7. Cucinotta D, Vanelli M. (2020).WHO Declares COVID-19 a Pandemic. Acta Biomed,19;91(1):157-160. doi: 10.23750/ abm.v91i1.9397. PMID: 32191675; PMCID: PMC7569573.
8. Dave Roos. (2020). How 5 of History's Worst Pandemics Finally Ended. https://www.history.com/news/pandemicsend-plague-cholera-black-death-smallpox

9. Dental Council of India (2020), Covid-19 Guidelines for Dental Colleges, Dental Students and Dental Professionals by Dental Council of India, New Delhi, India.http://dciindia.gov.in/Admin/NewsArc hives/DCI/Guidelines/on/COVID-19.pdf.

10. Elshazli RM, Kline A, Elgaml A, Aboutaleb MH, Salim MM, Omar M, Munshi R, Mankowski N, Hussein MH, Attia AS, Toraih EA, Settin A, Killackey M, Fawzy MS, Kandil E.( 2021). Gastroenterology manifestations and COVID-19 outcomes: A meta-analysis of 25,252 cohorts among the first and second waves. J Med Virol, 93(5):2740-2768. doi: 10.1002/jmv.26836. Epub 2021 Feb 23. PMID: 33527440; PMCID: PMC8014082.

11. Jyoti Prajapat, S, Rajesh Prajapat, Sowmya H K, Sanjeev B Khanagar, Prabhadevi C Maganur, \& Satish Vishwanathaiah. (2021). Teledentistry: The Need of An Hour During Pandemic COVID-19. Indian Journal of Forensic Medicine \& Toxicology, 15(2), 1706-1708. https://doi.org/10.37506/ijfmt.v15i2.14584

12. Parin Bhanushali, Farhin Katge, Shantanu Deshpande, Vamsi Krishna Chimata, Shilpa Shetty, Debapriya Pradhan, (2020). "COVID-19: Changing Trends and Its Impact on Future of Dentistry", International Journal of Dentistry, Article ID 8817424,6 pages, 2020. https://doi.org/10.1155/2020/8817424

13. Xian Peng, Xin Xu, Yuqing Li, Lei Cheng, Xuedong Zho and Biao Ren. (2020) Transmission routes of 2019-nCoV and controls in dental practice. Int $\mathbf{J}$ Oral Sci, 12(1):9.

14. Puneet Bhatnagar. (2020). What is the 'COVID-ified' future for dentists?.Mar $25^{\text {th }}$.

15. Sikka N, Willis J, Fitall E, Hall KK, Gale B. (2020). Telehealth and Patient Safety During the COVID-19 Response. Rockeville, MD: AHRQ PSNet [Serial online

"https://psnet.ahrq.gov/perspective/telehealt h-and-patient-safety-during...".

16. Singh N, Sultan A, Juneja A, Aggarwal I, Palkit T, Ohri T. (2020 ). Integration of teledentistry in oral health care during 
COVID-19 pandemic. Saint Int Dent J, 4:77-81.

17. Singh H, Kaur H, Singh K, Sen CK. (2020). Cutaneous Manifestations of COVID-19: A Systematic Review. Adv Wound Care (New Rochelle), 10(2):51-80. doi: 10.1089/ wound.2020.1309. Epub 2020 Oct 19. PMID: 33035150; PMCID: PMC8020517.

18. Rami Sommerstein, Christoph Andreas Fux, Danielle Vuichard-Gysin, Mohamed Abbas, Jonas Marschall, Carlo Balmelli, et al., (2020). Risk of SARS-CoV-2 transmission by aerosols, the rational use of masks, and protection of healthcare workers from COVID-19. Antimicrob Resist Infect Control,9(1):100.

19. Puneet Bhatnagar. (2020).What is the 'COVID-ified' future for dentists? https://www.dentistryiq.com/dentistry/articl e/14172811/what-is-the-covidified-futurefor-dentists.
20. Xu S, Li Y. (2020).Beware of the second wave of COVID-19. Lancet, 25;395 (10233):1321-1322. doi: 10.1016/S01406736(20)30845-X. Epub 2020 Apr 8. PMID: 32277876; PMCID: PMC7194658.

21. Zhao Q, Fang X, Pang Z, Zhang B, Liu H, Zhang F. (2020). COVID-19 and cutaneous manifestations: a systematic review. J Eur Acad Dermatol Venereol. Nov;34(11):25052510. doi: 10.1111/jdv.16778. Epub 2020 Aug 26. PMID: 32594572; PMCID: PMC7361780.

How to cite this article: Sriharsha P, Shaik N, Yadlapati S et.al. Decoding COVID-19: what dentist should know? International Journal of Science \& Healthcare Research. 2021; 6(4): 6470. DOI: https://doi.org/10.52403/ijshr. 20211010 